\section{MS35-O2 Diffuse scattering, spectroscopy and ab initio density functional theory}

Matthias J. Gutmann ${ }^{1}$

1. Rutherford Appleton Laboratory, ISIS Facility, Chilton Didcot, Oxfordshire OX11 0QX, United Kingdom

email: matthias.gutmann@stfc.ac.uk

Diffuse scattering in a crystal arises from any local deviation from long-range order and can be dynamic or static inorigin. Most of the time, phonons are the main cause and this is known as thermal diffuse scattering. Phonons can beobtained from density functional theory and examples will be shown, where such calculations are compared with diffusescattering from single crystals and phonon spectra from powdered samples. When using neutrons it is possible undercertain circumstances to observe phonon excitations directly in a diffraction pattern. This leads to rather peculiardiffuse scattering which breaks the symmetry of the diffuse scattering and an example of this will be shown [1].The second part will focus on benzil. Diffuse scattering in benzil has been the subject of a number of studies using X-rays and neutrons $[2,4]$. So far mostly Monte-Carlo modelling has been applied to the room-temperature phase [2]. Such a model has allowed least-squares refinement against joint X-ray and neutron data [3]. Here, we use an alternative approach to model the thermal diffuse scattering using ab-initio phonons. The study is extended to the diffuse scattering in the low temperature phase. The ab-initio models are compared to neutron spectroscopic data in the two phases leading to a complete assignment of the phonons. In a previous neutron experiment [4], interesting inelastic effects were observed, leading to peculiar modification of the diffuse scattering and ascribed to direct phonon excitations. However, this effect could not be simulated so far. Here, we employ a recently developed methodology to give a complete account of this effect [1].

[1] M. J. Gutmann, G. Graziano, S. Mukhopadhyay, and K. Refson, J. Appl. Cryst. 48, 1122 (2015).

[2] T. R. Welberry, D. J. Goossens, A. J. Edwards and W. I. F. David, Acta Cryst. A57, 101 (2001)

[3] D. J. Goossens, T. R. Welberry, A. P. Heerdegen, and M. J. Gutmann, Acta Cryst. A63, 30 (2007).

[4] T. R. Welberry, D. J. Goossens, W. I. F. David, M. J. Gutmann, M. J. Bull and A. P. Heerdegen, J. Appl. Cryst. 36, 1440 (2003)

\section{MS35-O3 Predicting Urea Crystal Shapes Grown from Solution with Molecular Dynamics Simulations \\ Zoran Bjelobrk ${ }^{1}$, Matteo Salvalaglio ${ }^{2}$, Michele Parrinello
Marco Mazzotti
Mar,}

1. Institute of Process Engineering, ETH Zürich, Switzerland

2. Department of Chemical Engineering, University College London, United Kingdom

3. Department of Chemistry and Applied Biosciences, ETH Zürich, Switzerland

4. Facoltà di Informatica, Istituto di Scienze Computazionali, Università della Svizzera Italiana, Switzerland

\section{email: zoranb@ethz.ch}

Crystallization is a common purification method for the production of active pharmaceutical ingredients and fine chemicals. The shape of crystalline particles influences downstream unit operations such as filtering and tableting, as well as physical and chemical properties such as solubility and bioavailability. Hence, control of the crystal shape is of crucial interest in the manufacturing of pharmaceutical compounds.

The macroscopic growth morphology of crystals is determined by the relative growth rates of the individual crystal faces [1]. Therefore, an atomistic description of the crystal surface dynamics, including the interaction of the crystal surface, solute, solvent and additives, allows for the prediction of crystal shapes grown from solution [2].

In this work, we examine the morphology of urea crystals grown from different solutions through the combination of molecular dynamics simulations and theory together with dedicated experiments $[2,3]$. The free energies of adsorption of the liquid phase molecules onto the particular crystal faces are evaluated with advanced simulation methods comprising well-tempered metadynamics and variationally enhanced sampling [4]. From the molecular dynamics simulations and theory we are able to predict the steady-state crystal habits of urea in dependence of solvent, additive concentration, and supersaturation.

[1] A. A. Chernov Sov. Phys. Crystallogr. 1962, 7, 728-730.

[2] M. Salvalaglio et al. Angew. Chem. Int. Ed. 2013, 52, 13369-13372.

[3] M. Salvalaglio et al. J. Am. Chem. Soc. 2012, 134, $177221-17233$.

[4] O. Valsson et al. Phys. Rev. Lett. 2014, 113, 090601.

Keywords: crystallization, molecular dynamics, urea

Keywords: diffuse scattering, neutron, X-ray 